\title{
Impact of Energy Depletion and Reliability on Wireless Sensor Network Connectivity
}

\author{
Jae-Joon Lee, Bhaskar Krishnamachari and C.-C. Jay Kuo \\ Department of Electrical Engineering, University of Southern California \\ Los Angeles, CA 90089-2564, USA, \\ E-mails: \{jaejlee, bkrishna\}@usc.edu, cckuo@sipi.usc.edu
}

\begin{abstract}
The impact of the non-uniform individual sensor node lifetime on the connectivity of a data gathering tree over time is studied in this research. The lifetime of sensor devices depends on the device failure rate and/or battery energy depletion, and surviving nodes may not preserve the uniform node density across the network as nodes age. We first examine the general node aging problem by considering the energy consumption rate and the node failure rate. The energy consumption rate in a data gathering tree is presented with or without data aggregation. The nodes in each hop level show a different energy depletion rates even with data aggregation, which is studied by mathematical analysis as well as simulation results. Then, the resulting non-uniform connectivity over time in a data gathering tree is examined with a node's survivor function. It is shown by mathematical analysis and simulation results that the node aging process has a significant impact on the connectivity as the hop distance increases.
\end{abstract}

Keywords: Aging, Energy Consumption, Reliability, Connectivity, Data Gathering

\section{INTRODUCTION}

Sensor networks consist of a large number of low-cost wireless sensor devices with limited battery energy. There are two factors that determine the sensor device lifetime: battery energy depletion and device failure. Energy consumption depends on the activities of each component in the sensor node device, which are mainly determined by the applications and network management schemes. Device reliability is affected by unexpected events. Device hardware and software components can fail due to either internal problems or external impacts. When thousands of sensor devices are deployed in an adversary environment, the normal function of a node cannot be guaranteed even before its battery energy get depleted. These individual node failures caused by either energy depletion or device failure result in a negative impact on network operations, including the sensing coverage of the area and the connectivity among nodes.

For many wireless sensor applications, it may not be necessary to preserve the connectivity between any two arbitrary end-points. For example, each node transmits the collected data to the sink along the data gathering tree in the data gathering application. For such an application, the connectivity between the target group of nodes and their sink is the most critical one, and should be preserved as much as possible during the entire network lifetime. The data gathering tree is constructed to provide the communication path between the sink (i.e. the root) and nodes that are the source. The most common data gathering construction scheme can be stated as follows. The sink initiates message broadcasting to the entire network and all receiving nodes select the parent node within its radio range among the nodes that forward the broadcast message from the sink [1]. When a sensor node has data to be sent to the sink, it forwards the data to the parent node in the data gathering tree. If the application allows data aggregation, each node can aggregate all the data from its children and sends the aggregated data toward the sink. Data aggregation reduces the amount of data transmitted in the data gathering tree, which saves the communication energy of forwarding nodes.

The node lifetime in a data gathering tree mainly depends on the energy consumption rate of the node. As discussed in many studies, including [2], battery energy is dominantly consumed by the communication function. In a data gathering tree, the largest amount of communication energy of a node is consumed in receiving the data from its child nodes and transmitting the data to its parent node. Since the number of children or descendants of each node is not identical, which determines the number of packets forwarded to each node, the battery energy 
consumption rates are different among nodes and the network cannot preserve the uniform node density across the network over time. The impact on the connectivity by this non-uniform energy depletion process and the node survivor analysis in a dynamic network over time are not well understood.

In this work, we examine the non-uniform characteristics of the node aging process by considering both energy depletion and reliability factors, and then analyze their impact on the connectivity in a data gathering tree. First, the energy consumption of nodes in a data gathering tree is discussed with or without data aggregation. We focus on the communication energy consumption in each hop distance from the sink and show the non-uniform energy depletion property among nodes based on the number of packets that each node forwards. Then, we provide a node survivor function that takes both energy depletion and reliability into account and analyze the connectivity model over time, which is dependent on the dynamic node aging behavior. With extensive simulations, we examine the non-uniform node aging property in a data gathering tree with different radio ranges and study the connectivity loss in the tree when the majority of nodes is still alive. Finally, we observe that the lower bound of connectivity presented here is tight as compared to the simulation result which presents the impact of the node reliability on the connectivity.

The rest of this paper is organized as follows. Previous work on connectivity, power consumption and data gathering is reviewed in Section 2. Energy consumption in a data gathering tree with or without data aggregation is discussed in Section 3. Section 4 presents the reliability model and the node survivor function and then analyzes the connectivity on a data gathering tree in each hop level and the lower bound of the connectivity model. Simulation results and the evaluation of the connectivity lower bound are given in Section 5. Finally, concluding remarks and future research directions are given in Section 6.

\section{REVIEW OF PREVIOUS WORK}

The connectivity of wireless networks has been studied by several research groups. Most of the studies focus on determining the radio range that ensures the connectivity between any two nodes in an uniformly distributed network, e.g. [3-5]. Gupta and Kumar [3] provided a theoretical analysis for the critical threshold of the radio range that guarantees the connectivity over randomly distributed nodes. Bettstetter [4] investigated the relation between connectivity and several underlying factors such as the radio range and the node degree in a random uniform distribution. The above two studies offer some node deployment guideline to ensure the desired connectivity. Shakkottai et al. [5] considered the node failure effect and provided a bound on the probability that all nodes are connected and all network area is covered. However, their analysis was mainly concerned with the full connectivity of all nodes (rather than the partial connectivity of the network) when nodes are uniformly distributed and the failure probability is also uniform across the network. Thus, they did not provide the connectivity change analysis over time due to node failure and energy depletion, under which the uniform node density property may not be preserved any longer.

The energy consumption model and the network lifetime analysis have been studied in $[6,7]$. Heinzelman et al. [6] provided a general energy consumption model for communication components in a sensor device. They considered energy consumption with the multi-hop as well as the direct communication mechanisms, and compared the lifetime of three communication schemes: direct, multi-hop, and cluster-based data gathering. The network lifetime is determined by energy depletion, which in turn depends on the number of packets that a node processes and its radio range. However, they did not consider data aggregation in the multi-hop communication case. Bhardwaj and Chandrakasan [7] discussed the upper bound of network lifetime by considering topology and data aggregation. They presented a feasible role assignment method, in which three roles - sensing, relaying, and aggregating - are assigned to nodes to derive the lifetime bound in a network of any arbitrary topology with data aggregation using the general energy consumption model. All these studies focused on the energy depletion instance of nodes and did not consider the dynamic node aging process and its impact on the connectivity, which is our work in this paper.

Data gathering schemes and the corresponding data aggregation analysis were discussed in $[1,8,9]$. Intanagonwiwat et al. [8] presented a data gathering scheme that provides the data centric routing with data aggregation. In their scheme, the sink sends out the interest for a specific type of data while the receiving nodes keep this information and forward the interest information to neighboring nodes. When the desired data are 
present in a node, the node sends the data towards the sink in the reverse path of interest propagation. The energy-saving aspect of data aggregation was examined by Krishnamachari et al. [9]. They provided several source models and analyzed the energy cost savings and delay resulted by data aggregation. Data gathering tree construction schemes were discussed by Zhou and Krishnamachari in [1]. In this work, sensor nodes select the parent node in a data gathering spanning tree using one of four parent selection methods. They compared these parent selection methods with several metrics, including the variance of the node degree, robustness, channel quality, the degree of data aggregation and latency of data gathering. We used the parent selection scheme given in [1] to analyze the node aging characteristics and the impact on the connectivity in a data gathering tree. Being different from the studies presented above, we examine the dynamic energy consumption rate in each node per hop level by considering the number of packets processed by a node based on the number of children and descendants in the tree.

\section{ENERGY CONSUMPTION IN DATA GATHERING TREES}

Battery energy consumption is the dominant factor in node aging when the network deployment environment is favorable and the device is highly reliable. There are several major operations done by sensor nodes, which consume the most of battery energy. They include: communication, sensing, and data processing. Sensor nodes participate in communications with various purposes depending on applications and network management schemes. In most of applications, data gathering imposes the most critical communication requirement. For example, in the environment monitoring application, the data collected by a sensor node should be forwarded to the sink that acts as the bridge between the sensor network and the observer. The following three factors contribute the main communication energy consumption in the data gathering process.

1. Participants of a data gathering tree include nodes in the forwarding path. They are either nodes that have data to send or cluster heads that collect and process data from the nodes in the cluster.

2. If the sensor network performs in-network processing using a cluster to reduce the amount of data to be forwarded to the sink, the communication between the cluster head and its clustering nodes provides the second power consumption source.

3. The third factor that demands energy consumption is the exchange of control messages to construct and manage the network infrastructure. This includes the establishment and management of data gathering paths and clusters. Furthermore, some networks would require node localization, time synchronization and calibration.

The sensing and data processing tasks also demand energy. However, the amount of required energy consumption is less than that for communication [2]. In this section, we focus on the energy consumption in a data gathering tree. We discuss and analyze factors that affect the energy consumption in a tree by considering the number of packets processed by a node during data gathering.

\subsection{Energy Consumption for Data Gathering Communication}

A data gathering tree is formed such that all deployed sensor nodes are connected to a sink. Each node has a parent node which provides the forwarding path to the sink and its own children. A node receives data only from its children and forwards data only to its parent for the data gathering purpose.

\subsubsection{Energy Consumption Analysis}

Communication energy consumption in data gathering paths depends on the number of packets that a forwarding node receives and transmits. Thus, we can calculate the data gathering energy consumption for node $i$ at time $t$ as

$$
E_{i}^{G}(t)=E^{R} b_{i}^{R}(t)+E^{T} b_{i}^{T}(t),
$$

where $E^{R}$ and $E^{T}$ denote the amounts of energy required to receive and transmit a bit, and $b_{i}^{R}(t)$ and $b_{i}^{T}(t)$ are the numbers of bits that node $i$ receives and transmits at time $t$, respectively. $E^{T}$ includes the energy required for an amplifier to transmit a bit over a certain distance. 


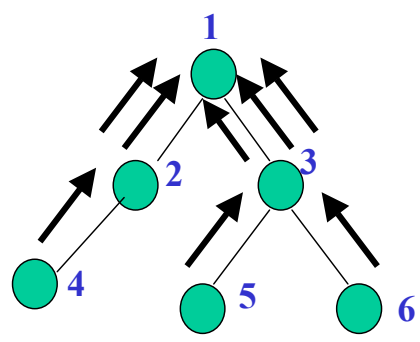

(a)

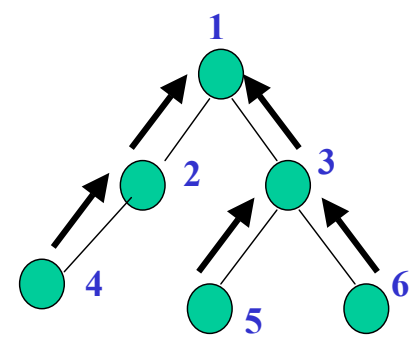

(b)

Figure 1. Illustration of the packet forwarding mechanism in a data aggregation tree: (a) without data aggregation and (b) with data aggregation.

By assuming that every node sends one packet to the sink per round time, the numbers of packets received and transmitted by a node can be written, respectively, as

$$
b_{i}^{R}(t)= \begin{cases}c N_{i}^{c}(t), & \text { with data aggregation, } \\ c N_{i}^{d}(t), & \text { without data aggregation, }\end{cases}
$$

and

$$
b_{i}^{T}(t)= \begin{cases}c, & \text { with data aggregation, } \\ c\left(N_{i}^{d}(t)+1\right), & \text { without data aggregation, }\end{cases}
$$

where $N_{i}^{d}(t)$ is the number of node $i$ 's descendants that sent the data to the sink, and $N_{i}^{c}(t)$ is the number of node $i$ 's children that have the source descendants, and $c$ is the averaged number of bits per packet. For the data aggregation case, we consider the perfect data aggregation scheme where the data from multiple sources can be aggregated to generate a single result (e.g., MIN, MAX, COUNT, SUM) in order to compare the two extreme energy consumption situations.

In Fig. 1, we illustrate the packet forwarding mechanism in a data gathering tree with or without data aggregation. For the case without data aggregation as shown in Fig. 1(a), all packets from the descendant nodes are forwarded to the sink along the tree. For the case with data aggregation as shown in Fig. 1(b), a parent node processes packets received from the children rather than just forwarding them. If the data aggregation operation is performed in a node, it is often that a single packet is generated by combining its own data with the data from its children. Accordingly, the number of packets that a node in a data gathering tree receives per round time is same as the number of its children that send out packets as shown in Fig. 1(b).

In a dynamic data gathering tree, the number of children and descendants of a node change over time. Since the tree structure is dynamically reconfigured due to node's death or disconnection, it results in the increase or decrease of communication burden for other nodes. For example, in Fig. 1(a), when node 3 dies, if node 2 is within the radio range of nodes 5 and 6 , node 2 is selected as the new parent of nodes 5 and 6 . Thus, the number of packets forwarded to node 2 per round time increases from 1 to 3 without data aggregation, which results in higher energy consumption by three folds. However, the number of packets forwarded to node 1 reduces from 5 to 4 , which decreases the energy consumption rate of node 1 .

\subsubsection{Numbers of Children and Descendants}

The energy consumption rate of a forwarding node in a data gathering tree depends on the number of children (with data aggregation) or the number of descendants (without data aggregation) as discussed in Section 3.1.1. When nodes are uniformly distributed at random and the sink is located at the center of the network area, the average number of children and descendants can be estimated by calculating the area of each hop level in the network. The hop level is defined to be the hop distance from the sink. If the radio range forms a complete circle, then $l$ hop level's area is $\left((l+1)^{2}-l^{2}\right) \pi r^{2}=(2 l-1) \pi r^{2}$. The ratio of the number of nodes in each hop 
would be same as the ratio of the area for a uniform and dense node distribution. If all nodes participate in forming the data gathering tree where the sink is the root, the average number of children of a node at hop level $l$ would be $1+2 /(2 l-1)$. This shows that the nodes at the first hop level have more children than nodes at lower levels. Thus, the energy consumption rates of nodes in a data gathering tree are not uniform even with perfect data aggregation. For the non-data aggregation case, the number of descendants of a node at the lth hop level is determined by

$$
\sum_{i=l}^{L-1}\left(\prod_{j=l}^{i}\left(1+\frac{2}{2 j-1}\right)\right)
$$

where $L$ is the maximum hop distance from the sink.

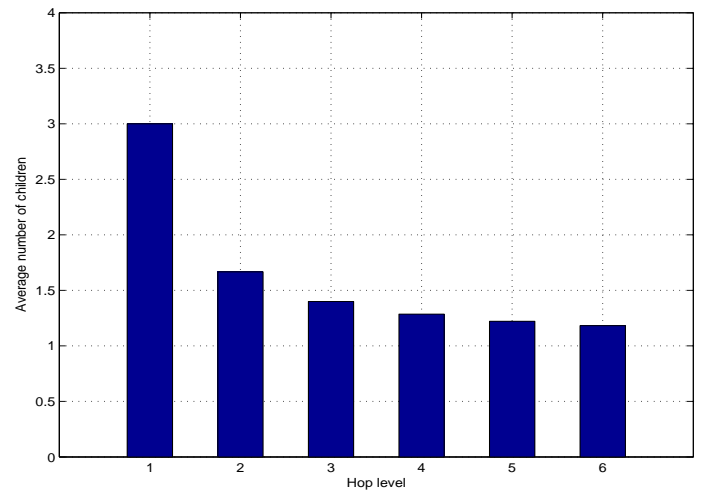

(a) The number of children

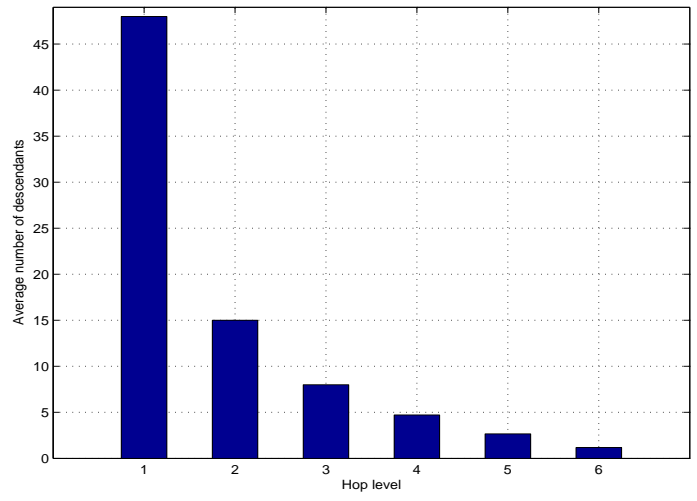

(b) The number of descendants

Figure 2. The number of children and descendants in each hop level when the maximum hop distance is 7 .

As shown Fig. 2, the number of children and descendants of a node at the first hop is much larger than that at other hops. The average number of children and the ratio of the descendant numbers at two consecutive hop levels remain the same regardless of the network size. In contrast, the number of descendants depends on the network size, which can be represented as the maximum hop counts from the sink.

\subsection{Total Energy Consumption Analysis}

Besides the communication energy consumption, there are other energy consumption activities done by nodes in sensor networks, which include data processing and sensing operations. They are addressed separately below.

- In-network processing reduces the number of packets to be sent to the sink by processing and aggregating the data within a cluster or along the way to the sink. This can save the overall energy consumption in the network. For cluster-based in-network processing, the communication and data processing burden of a cluster head is higher than other nodes, which causes faster energy depletion. There are two main data processing operations demanding power consumption; namely, the processing of sensed raw data and the data aggregation processing.

- As to sensing operations, the degree of energy consumption can be quite different among different types of sensors used for various applications.

In this paper, we consider two other energy consumptions operations besides communications. One is the data processing energy consumption denoted by $E^{D}$ and the other is the sensor energy consumption denoted by $E^{S}$.

To summarize, we consider three main energy consumption operations in modelling the energy consumption of each node: (1) data gathering communication, (2) sensing and (3) data processing. Let $E_{i}$ be the initial 
battery energy at time 0 and $E_{i}(t)$ be the remaining energy at time $t$ for node $i$. Then, $E_{i}(t)$ is given by

$$
E_{i}(t)=E_{i}-\sum_{k=1}^{t}\left(E_{i}^{G}(k)+E_{i}^{S}(k)+E_{i}^{D}(k)\right) .
$$

If $E^{S}$ and $E^{D}$ are identical in all nodes over time, then the energy consumption rate of a node is mainly dependent on $E_{i}^{G}$, which represents the number of packets forwarded by the node. As nodes age over time, the energy consumption rate of each node dynamically changes over time due to other node's battery energy depletion or device failure as discussed in Sect. 3.1.1.

\section{NODE SURVIVOR FUNCTION AND CONNECTIVITY ANALYSIS}

In this section, we first discuss the node survivor function by considering effects of battery energy depletion and device reliability. Then, the connectivity on a dynamic data gathering tree at each hop level and the lower bound of the connectivity model are analyzed.

\subsection{Reliability}

The internal hardware or software problems of a sensor device can result in its failure of proper functioning. In addition, the external environment e.g., rain, lightening and other physical impacts, may cause a negative impact on the sensor device operation. Thus, the survivor function of a sensor device depends not only on the battery energy depletion but also the device failure rate. Energy depletion would be the dominant factor to consider if the sensor device is highly reliable. However, the low cost sensor device is prone to unexpected failure as well.

The device failure time distribution has a diverse range of shapes and scales among different device types and deployment environments. We use the classical Weibull distribution [10] to model the sensor device lifetime of various shapes and scales. The probability density function of the Weibull distribution is of the following form:

$$
f(t)=\frac{\beta}{\eta}\left(\frac{t}{\eta}\right)^{\beta-1} e^{-\left(\frac{t}{\eta}\right)^{\beta}}
$$

where $\beta$ is a positive shape parameter, $\eta$ the scale parameter and $t$ the time index. Then, the reliability function, which is the probability that node $i$ is functioning normally at time $t$, can be expressed as

$$
R_{i}(t)=e^{-\left(\frac{t}{\eta_{i}}\right)^{\beta_{i}}}
$$

which is the complement of the cumulative distribution function $F(t)$ of the Weibull density function given in (5).

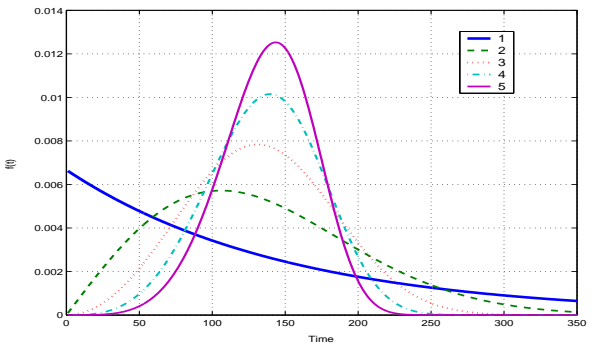

(a) $\beta$ from 1 to 5 with $\eta=150$

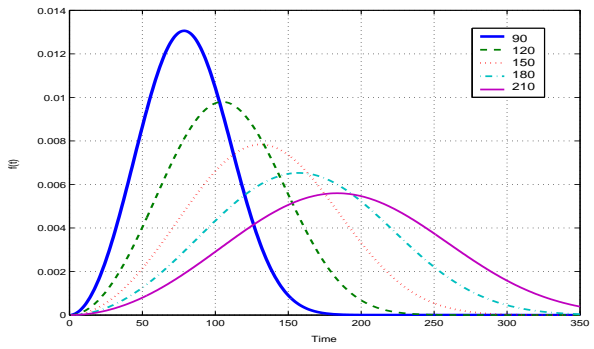

(b) $\eta$ from 90 to 210 with $\beta=3$

Figure 3. The probability density functions of the Weibull distribution.

Figs. 3 and 4 show the probability density function and the reliability function of the Weibull distribution, respectively, with several shape parameters $\beta$ and scale parameters $\eta$. In these subfigures, $\eta$ is set to 150 with different $\beta$ values, and $\beta$ is set to 3 with different $\eta$ values for comparison. 


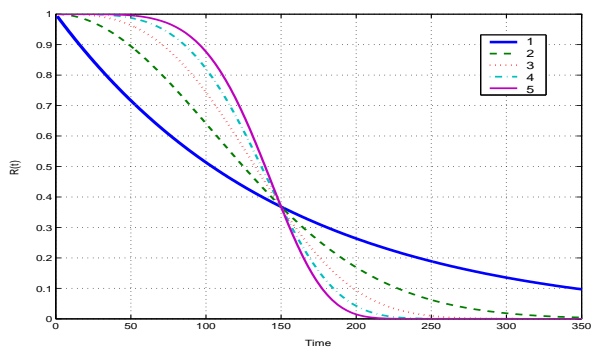

(a) $\beta$ from 1 to 5 with $\eta=150$

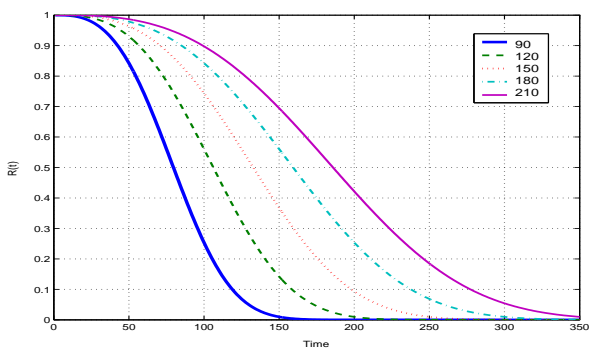

(b) $\eta$ from 90 to 210 with $\beta=3$

Figure 4. The reliability functions for the Weibull distribution

If all deployed sensor devices are the same and under the same environment, $R_{i}(t)$ would be identical for all nodes. On the other hand, heterogeneous devices may have different scales and shapes in their lifetime distributions. For example, we can deploy two different types of sensor devices such that the highly reliable device equipped with more battery energy, which is also more expensive, can be used as the cluster head. The more reliable device has a larger scale parameter.

\subsection{Node Survivor Function}

The survivor function of node $i$, denoted by $S_{i}(t)$, is defined to be the probability that node $i$ is functioning at time $t$. The survivor function depends on battery energy depletion as well as the device failure rate. In the following discussion, we follow the energy consumption model of each node as discussed in Sect. 3 for battery energy depletion, which is mainly dependent on the number of children or descendants. Furthermore, we follow the model given in Sect. 4.1 for the reliability of sensor nodes. Based on our above discussion, the survivor function $S_{i}(t)$ can be written as

$$
S_{i}(t)=1, \quad t=0 .
$$

and

$$
S_{i}(t)= \begin{cases}0, & \text { if } S_{i}(t-1)=0 \text { or } E_{i}(t)<0 \\ R_{i}(t), & \text { otherwise }\end{cases}
$$

for $t>0$. The survivor function is nonincreasing and, once it becomes zero, it stays zero, which means that the sensor device is not reparable or the battery is not replaceable. The interesting observation is that nodes at different hop levels in a data gathering tree have a different survivor function due to different energy depletion rates.

\subsection{Connectivity Analysis in Data Aggregation Trees}

The connectivity of a dynamic data gathering tree with non-uniform node survivor functions is analyzed here. Following [1], nodes locally construct a data gathering tree that establishes the communication paths from each sensor device to a sink by selecting a parent node in the upper hop level. In a dynamic data gathering tree, if a parent node dies or has no connection to the sink, then each child node selects a new parent node among candidate parent nodes that are still functioning. As the radio range increases, the node would have a larger number of candidate parents, which increases the connectivity in the presence of other node's death.

Let $C_{i}^{l}(t)$ be the event that node $i$ with an $l$-hop distance from the sink on the data gathering tree is connected to the sink at time $t$ and $A_{i}^{l}(t)$ be the event that node $i$ at the $l$ th hop level is alive and functioning at time $t$. We use $p_{i}^{l-1}$ to refer to any candidate ascendent nodes at hop level $l-1$ of node $i$ in a dynamic data gathering tree. Then, we have

$$
\operatorname{Pr}\left(C_{i}^{l}(t)\right)= \begin{cases}\operatorname{Pr}\left(A_{i}^{1}(t)\right), & l=1 \\ \operatorname{Pr}\left(\bigcup C_{p_{i}^{l-1}}^{l-1}(t)\right) \operatorname{Pr}\left(A_{i}^{l}(t)\right), & l \geq 2\end{cases}
$$


As given in (7), the probability that a node at a distance of $l$ hops away from the sink is connected to the sink is equal to the probability that any of its candidate parents is connected and the node is alive. The lower bound of (7) is the case where there is only one available parent for all nodes in the forwarding path:

$$
\operatorname{Pr}\left(C_{i}^{l}(t)\right) \geq\left(\prod_{k=1}^{l-1} \operatorname{Pr}\left(A_{p_{i}^{k}}^{k}(t)\right)\right) \operatorname{Pr}\left(A_{i}^{l}(t)\right), \quad l>1,
$$

where $\operatorname{Pr}\left(A_{i}^{l}(t)\right)$ is equal to $S_{i}^{l}(t)$, which is the survivor function of node $i$ at the $l$ th hop level as discussed in Sect. 4.2. Then, we can derive the connectivity probability at each hop level with the survivor function. In a short radio range, where the number of neighbors is less than around 12, this lower bound shows a tight bound to the actual connectivity probability in a data gathering tree. We provide computer simulation results in the next section to demonstrate this point.

\section{COMPUTER SIMULATION RESULTS}

The effect of energy depletion and device failure on the connectivity in a data gathering tree is examined with extensive computer simulations. In the simulation environment, 400 nodes are uniformly distributed at random and the sink is located at the center of the network area as shown in Fig. 5. All simulation results are obtained as the average of 10 different random node deployments. After a random node deployment, a data gathering tree is constructed with the sink as the root. In the construction of the data gathering tree, each node selects the nearest node in the upper hop level as its parent among the candidate parents [1]. If a parent node dies or becomes disconnected from the tree, the node selects a new parent among its candidate parent nodes that are still functioning.

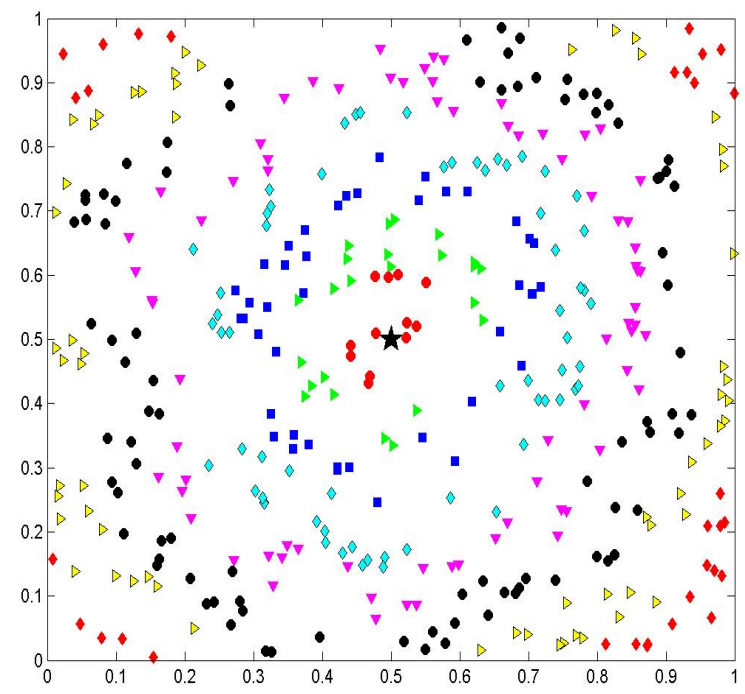

Figure 5. The illustration of a random node deployment.

We conducted the simulation with 3 different radio ranges to study the effect of the number of neighbors within the radio range on energy depletion and the connectivity of nodes at each hop level. The radio range is represented as the average number of neighbors and those are 8,12, and 18. Each radio range has a different average number of candidate parents, which are $2.4,3.4$, and 4.5 , respectively. In this simulation, we examine the energy depletion effect with and without data aggregation, the energy depletion impact on connectivity, and the reliability on connectivity at each hop level with a different radio range. 


\subsection{Data Aggregation versus Non-Data Aggregation}

Fig. 6 shows the energy depletion rate of nodes at each hop level with and without data aggregation. In this simulation, we use the radio range 12 for both cases. As shown in the figure, nodes at the first hop without data aggregation consume their energy much faster than the case with aggregation. This is due to the fact that the energy consumption rate without data aggregation is much higher than that with data aggregation due to the different numbers of packets forwarded in a tree as discussed in Sect. 3.1.2. Especially, the communication burden of the first hop nodes without data aggregation is much more than that with data aggregation. This results in early battery energy depletion for the first hop nodes. Thus, the data aggregation scheme enhances not only the node lifetime but also the overall connectivity to the sink since it reduces the large difference of the communication burden for nodes at different hop levels as shown in Fig. 6. However, even with data aggregation, we observe that the non-uniform energy depletion is consistent with the mathematical analysis given in Sect. 3.1.2.

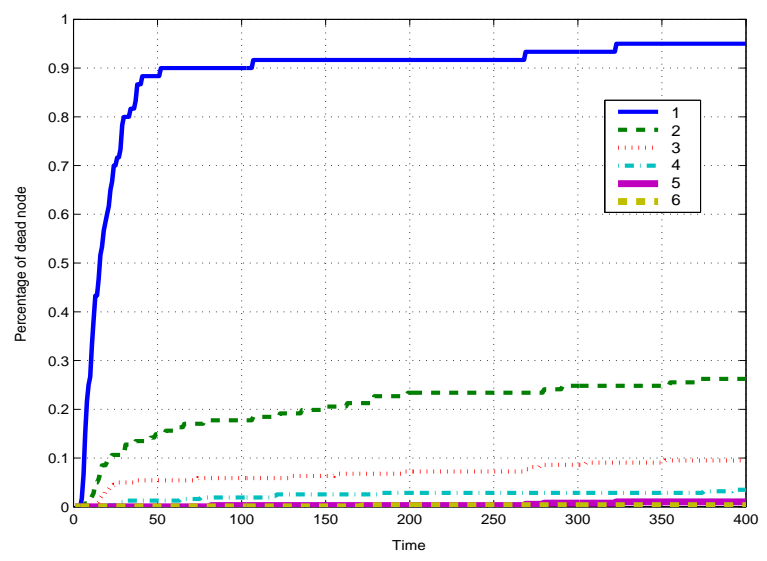

(a) without data aggregation

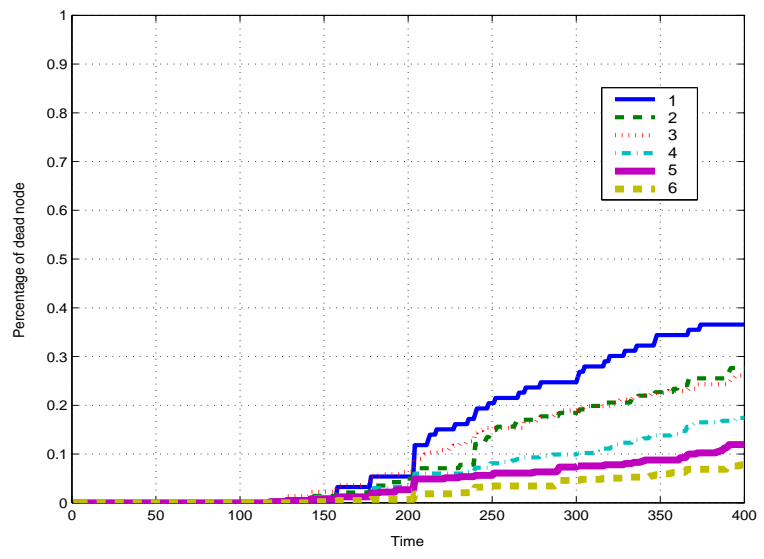

(b) with data aggregation

Figure 6. Energy depletion as a function of time with and without data aggregation.

\subsection{Radio Range Effect}

Fig. 7 shows the percentage of dead nodes over time at each hop level with different radio ranges. As discussed in Sect. 3.1.2, theoretically, the average number of children at each hop level remains the same regardless of the radio range. This results in a similar power depletion rate for different radio ranges. However, we see that the first hop node with an average of 8 neighbors as shown in Fig. 7(a) has a lower percentage of dead nodes (or a slower energy depletion rate) than those with a longer radio range as shown in Figs. 7(b) and (c). In contrast, the energy depletion rates of nodes at other hop levels are similar for different radio ranges. The reason for a slower energy depletion rate at the first hop nodes is the faster disconnection of these nodes. A large number of disconnection decreases the number of packets forwarded to these nodes and reduces their energy consumption rate.

The connectivity loss becomes bigger as the radio range gets shorter due to a smaller number of candidate parents as shown in Fig. 8. Since the number of neighbors and candidate parents are smaller with a shorter radio range, if the current parent to the sink dies, the node has a lower chance to recover the connection to the sink. Fig. 8(a) shows a drastic decrease of connectivity even when a high percentage of nodes are still alive. 80 $\%$ of node disconnection is observed with only less than $5 \%$ node dead. When the radio range is longer, the connectivity decreases almost linearly as shown in Fig. 8 (c).

\subsection{Connectivity at Each Hop Level}

The number of connected nodes at each hop level over time is shown in Fig. 9, where only the first 6 hop levels are shown to compare the effect of different radio ranges. Please note that the ratio of node numbers between 


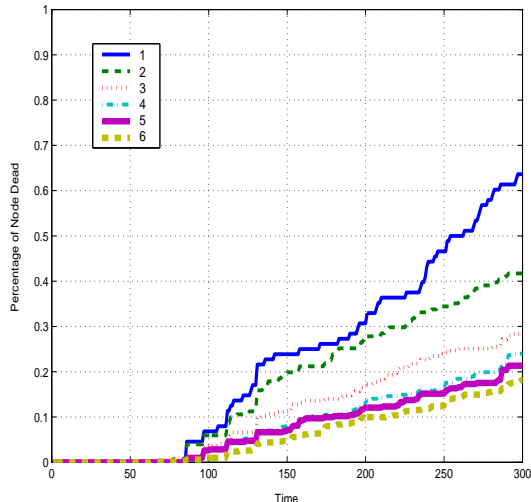

(a) Avg. number of neighbors: 8

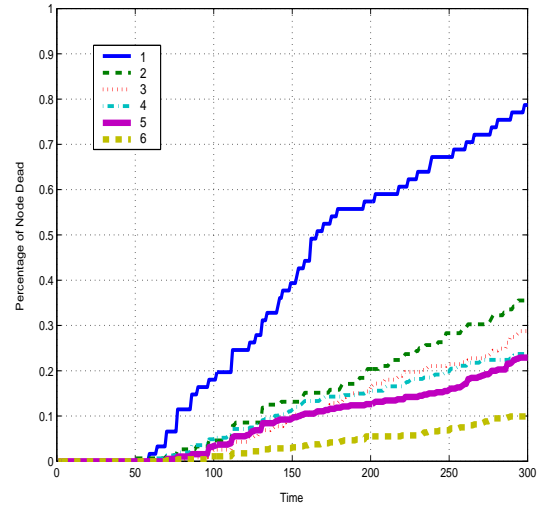

(b) Avg. number of neighbors: 12

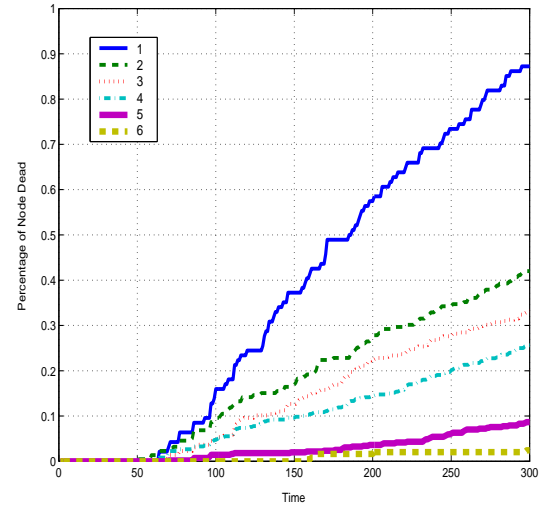

(c) Avg. number of neighbors: 18

Figure 7. The power depletion rate as a function of time for nodes at different hop levels.

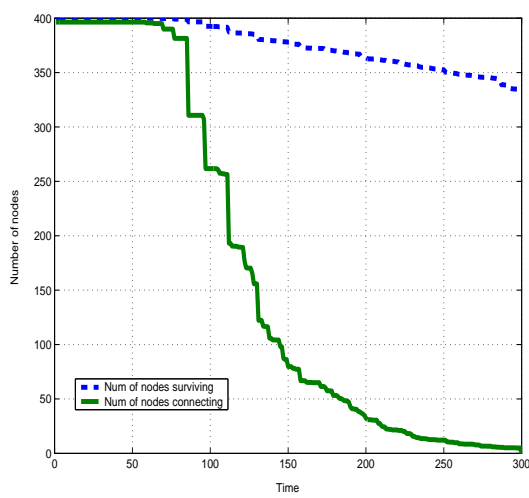

(a) Avg. number of neighbors: 8

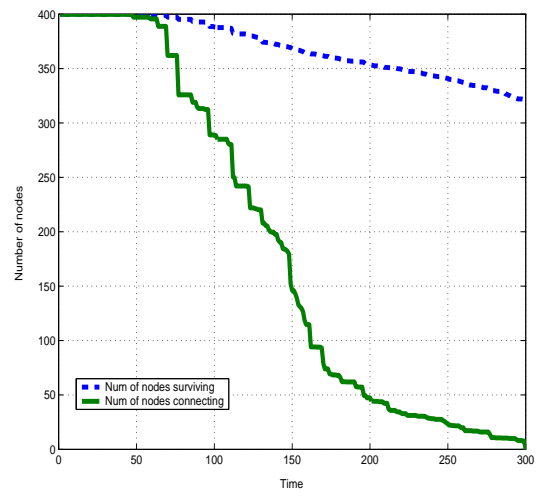

(b) Avg. number of neighbors: 12

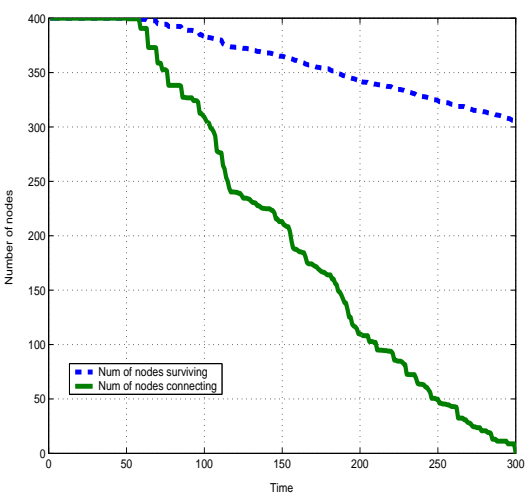

(c) Avg. number of neighbors: 18

Figure 8. The numbers of surviving nodes and connected nodes as a function of time.

two consecutive hop levels is similar for different radio ranges as discussed in Sect. 3.1.2. The main difference is the number of nodes at each hop level. Since the nodes in a shorter radio range rely on a smaller number of first hop nodes to connect to the sink, the connectivity loss rate is much faster than the longer radio range case. In addition, a small number of candidate parents results in a faster connectivity loss rate of nodes at lower hop levels.

The percentage of connected nodes at different hop levels over time is shown in Fig. 10. We see that these curves have a larger gap if the average number of neighbors is lower. Since a smaller number of neighbors limits the recovery of the data gathering tree, when a parent node fails, the connectivity at lower level hops decreases fast. The disconnection of lower hop level nodes reduces the communication burden of the first hop nodes, which enhances the lifetime of the remaining first hop nodes. As the number of neighbors increases with a larger radio range, the data gathering tree can be recovered with a better chance. Thus, the percentage of node connections at each hop level has a similar decrease rate over time as shown in Fig. 10(c).

We can compare the overall connectivity loss rate in Fig. 8 with the connectivity at each hop level in Fig. 10. Since the majority of nodes reside in a longer hop distance from the sink, we see that the overall connectivity loss rate follows the connectivity of nodes in a longer hop distance. 


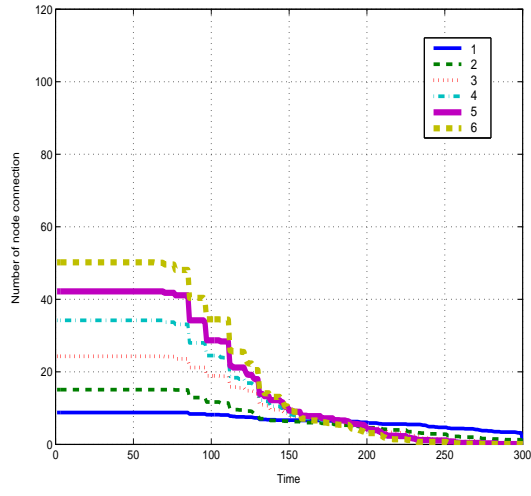

(a) Avg. number of neighbors: 8

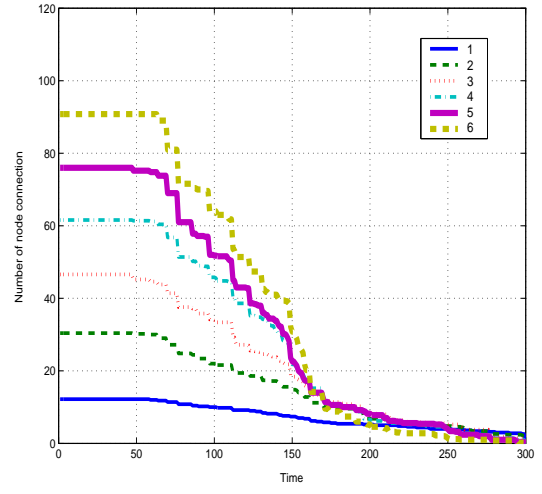

(b) Avg. number of neighbors: 12

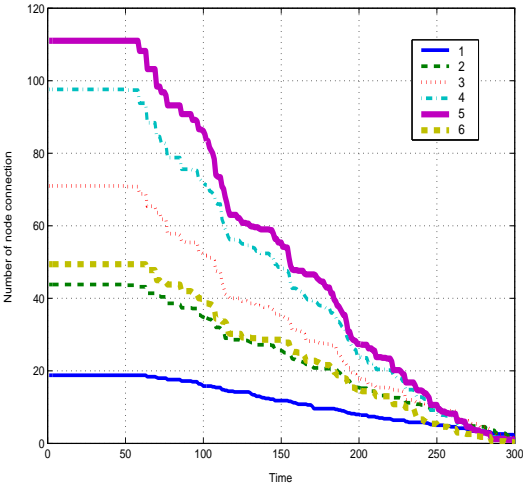

(c) Avg. number of neighbors: 18

Figure 9. The number of connected nodes at each hop level over time.

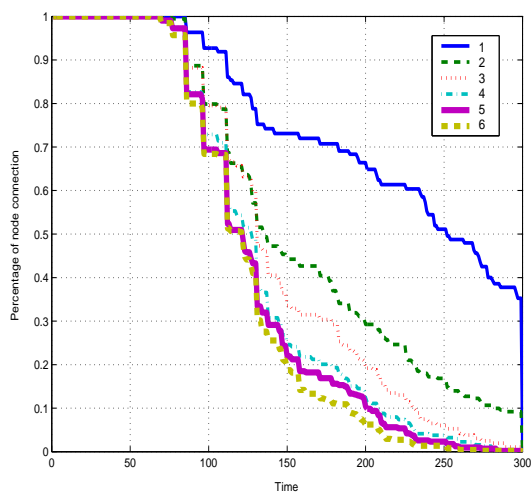

(a) Avg. number of neighbors: 8

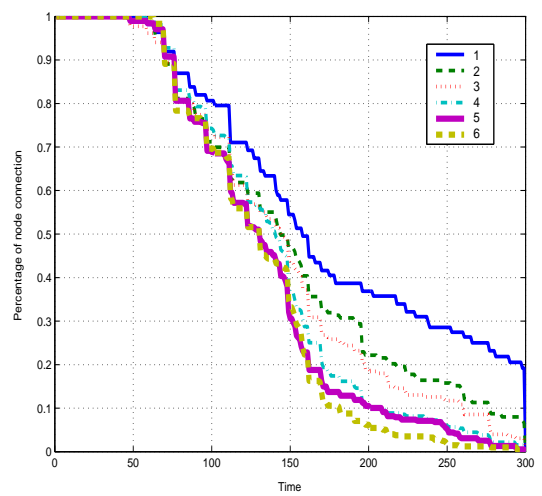

(b) Avg. number of neighbors: 12

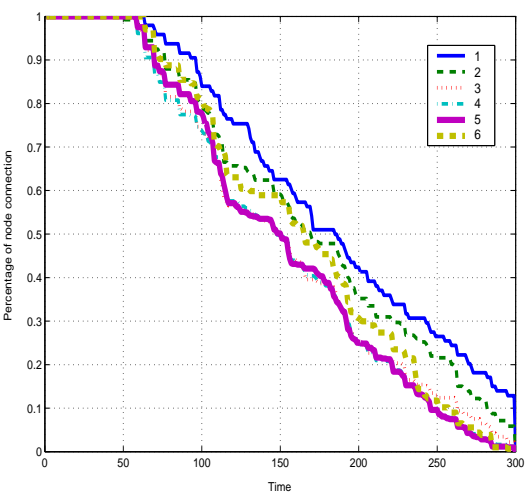

(c) Avg. number of neighbors: 18

Figure 10. The percentages of node connections at each hop level over time.

\subsection{Reliability effect on Connectivity in each hop level}

The analytical lower bound model derived in Sect. 4.3 is compared to the simulation result in Fig. 11. All nodes follow the identical reliability function discussed in Sect. 4.1 with $\eta=150$ and $\beta=3$ and have 8 neighbors on the average. The lower bound as given by (8), i.e. the probability that a node in each hop level is connected to the sink, is shown in Fig. 11(a). The simulation results are shown in Fig. 11(b). We observe that the simulation and analytical results match quite well. In addition, as the hop distance from the sink increases, the connectivity rapidly decreases even with a small number of node failures. Even with this uniform device failure model, the impact on the connectivity of nodes at a longer hop distance is critical.

\section{CONCLUSION AND FUTURE WORK}

The energy consumption issue in a dynamic data gathering tree with and without data aggregation was studied in this research. The reliability model was incorporated in deriving the sensor node survivor function. Finally, the impact of node aging on connectivity in a data gathering tree was examined with theoretical analysis as well as simulation results. When the node has a reasonable radio range with its average number of neighbors equal to 8 , we observed a significant connectivity loss even when most nodes are still alive.

From this study, we have the following observations. Even with the perfect data aggregation scheme adopted, the node aging phenomenon is not uniform among different hop levels due to the different ratio of children nodes in each hop. This has a significant impact on the connectivity of the data gathering tree. Thus, uniform 


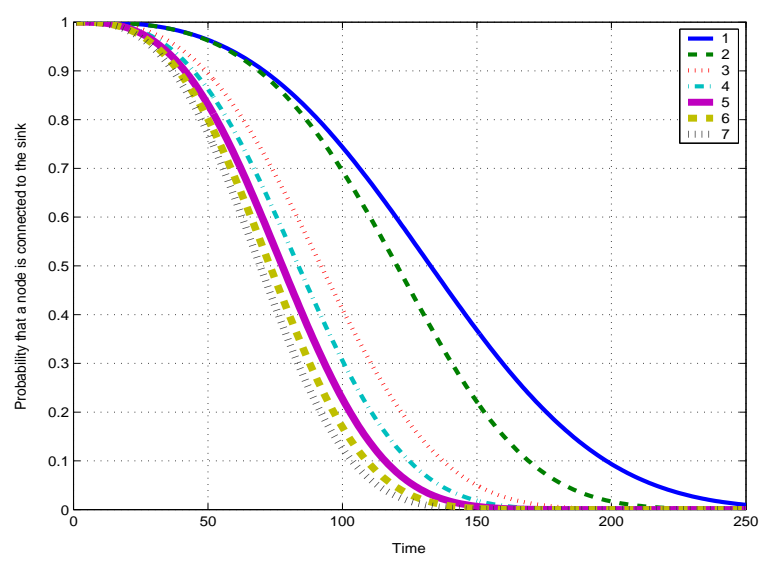

(a) Analytical lower bound

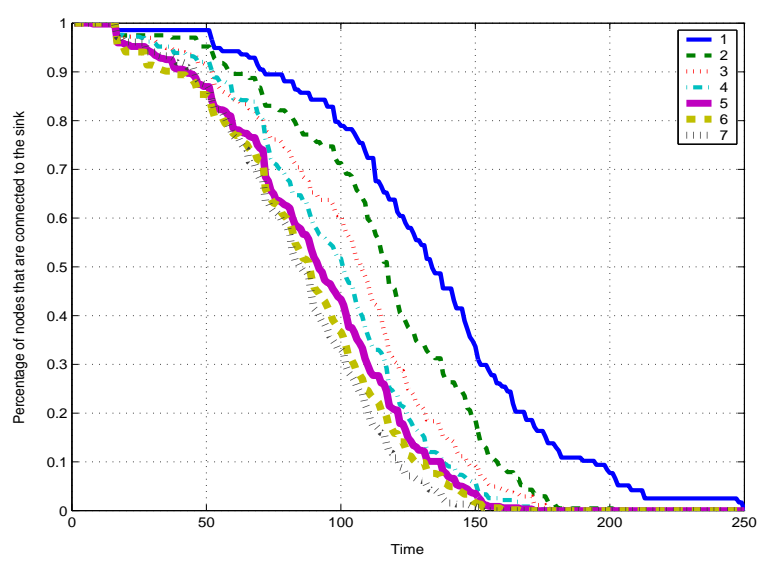

(b) Simulation result

Figure 11. The reliability effect on connectivity of nodes at different hop levels

energy depletion at each hop level is desirable to maximize the actual network lifetime. The understanding of the dynamic energy consumption behavior may help provide load balancing among nodes by assigning the different roles to nodes. The deployment of a small number of highly capable nodes (e.g., multiple sinks) will reduce the energy consumption of nodes and the maximum hop distance from the multiple sinks, which will enhance the connectivity of the network over time.

We intend to extend this work along the following directions. We will provide more concrete analytical models for energy consumption in a dynamic data gathering tree in addition to the tighter bound for the connectivity in a data gathering tree. We will also consider heterogeneous sensor device deployment to analyze the effect on node aging and connectivity.

\section{REFERENCES}

1. C. Zhou and B. Krishnamachari, "Localized topology generation mechanisms for self-configuring sensor networks," in Proc. IEEE GLOBECOM'03, 2003.

2. V. Raghunathan, C. Schurgers, S. Park, and M. B. Srivastava, "Energy-aware wireless microsensor networks," IEEE Signal Processing Magazine 19, pp. 40-50, 2002.

3. P. Gupta and P. R. Kumar, "Critical power for asymptotic connectivity in wireless network," in Stochastic Analysis, Control, Optimization and Applications, pp. 547-566, Birkhauser, Boston, 1998.

4. C. Bettstetter, "On the minimum node degree and connectivity of a wireless multihop network," in Proc. ACM MOBIHOC'02, 2002.

5. S.Shakkottai, R. Srikant, and N. Shroff, "Unreliable sensor grids: Coverage, connectivity and diameter," in Proc. IEEE INFOCOM'03, 2003.

6. W. Heinzelman, A. Chandrakasan, and H. Balakrishnan, "Energy-efficient routing protocols for wireless microsensor networks," in Proc. HICSS'00, 2000.

7. M. Bhardwaj and A. Chandrakasan, "Bounding the lifetime of sensor networks via optimal role assignments," in Proc. IEEE INFOCOM'02, 2002.

8. C. Intanagonwiwat, R. Govindan, and D. Estrin, "Directed diffusion: A scalable and robust communication paradigm for sensor networks," in Proc. ACM MOBICOM'00, 2000.

9. B. Krishnamachari, D. Estrin, and S. Wicker, "The impact of data aggregation in wireless sensor networks," in International Workshop on Distributed Event-Based Systems, 2002.

10. L. M. Leemis, Reliability: Probabilistic Models and Statistical Methods, Prentice-Hall, Englewood Cliffs, NJ, 1995. 